\title{
SUBHARMONIC FUNCTIONS AND SURFACES OF NEGATIVE CURVATURE*
}

BY

\author{
E. F. BECKENBACH† AND T. RADO
}

\section{INTRODUCTION}

0.1. Given a piece of surface in general parametric representation

$$
x=x(u, v), y=y(u, v), z=z(u, v),
$$

the Gauss curvature $K$ of the surface is given by the familiar formula $\ddagger$

$$
\begin{aligned}
W^{4} K=\left|\begin{array}{ccc}
\left(-\frac{1}{2} G_{u u}+F_{u v}-\frac{1}{2} E_{v v}\right) & \frac{1}{2} E_{u} & \left(F_{u}-\frac{1}{2} E_{v}\right) \\
\left(F_{v}-\frac{1}{2} G_{u}\right) & E & F \\
\frac{1}{2} G_{v} & F & G
\end{array}\right| \\
-\left|\begin{array}{ccc}
0 & \frac{1}{2} E_{v} & \frac{1}{2} G_{u} \\
\frac{1}{2} E_{v} & E & F \\
\frac{1}{2} G_{u} & F & G
\end{array}\right|,
\end{aligned}
$$

where $E, F, G$ are the first fundamental quantities:

$$
E=x_{u}{ }^{2}+y_{u}{ }^{2}+z_{u}{ }^{2}, F=x_{u} x_{v}+y_{u} y_{v}+z_{u} z_{v}, G=x_{v}{ }^{2}+y_{v}{ }^{2}+z_{v}{ }^{2},
$$

and $W^{2}=E G-F^{2}$. As is usual in differential geometry, we assume throughout this paper that $W \neq 0$ for the representations to be considered.

Suppose now that the surface is given in an isothermic representation; that is to say, suppose that $E=G, F=0$. Put $E=G=\lambda(u, v)$. The assumption $W \neq 0$ is then equivalent to $\lambda(u, v)>0$. The above formula for $K$ reduces to the form

where $\Delta$ is the symbol

$$
K=\frac{1}{2 \lambda^{3}}\left(\dot{\lambda_{u}^{2}}+\lambda_{v}^{2}-\lambda \Delta \lambda\right),
$$

$$
\Delta=\frac{\partial^{2}}{\partial u^{2}}+\frac{\partial^{2}}{\partial v^{2}} .
$$

0.2. By computation it follows that

* Presented to the Society, April 14,1933, under the title On the isoperimetric inequality; received by the editors February 16, 1933.

$\dagger$ National Research Fellow.

$\ddagger$ See W. Blaschke, Differentialgeometrie, Berlin, J. Springer, 1930, p. 93. 
Hence, we have the formula*

$$
\Delta \log \lambda=\frac{\lambda \Delta \lambda-\left(\lambda_{u}{ }^{2}+\lambda_{v}{ }^{2}\right)}{\lambda^{2}} .
$$

$$
K=-\frac{1}{2 \lambda} \Delta \log \lambda
$$

Consequently, if $K \leqq 0$ on our surface, then $\Delta \log \lambda \geqq 0$, that is to say, $\log \lambda$ is subharmonic in the terminology of $F$. Riesz. $\dagger$ Conversely, if $\log \lambda$ is subharmonic, then $K \leqq 0$ on the surface.

This relation between subharmonic functions and surfaces of negative curvature suggests geometrical applications of the theory of subharmonic functions. On the other hand, the geometrical interpretation suggests questions concerning subharmonic functions. The purpose of this paper is to present a few results which we have obtained in this way.

0.3. One of our geometrical results is concerned with the isoperimetric inequality. Among all simply-connected plane regions whose boundaries are rectifiable and have a given length $l$, the circle has the maximum area. This fact may also be stated as follows: if $a$ is the area and $l$ the length of the boundary of a simply-connected plane region, then $a$ and $l$ satisfy the isoperimetric inequality $a \leqq l^{2} /(4 \pi)$. Carleman proved that this same inequality holds for every simply-connected rectifiable piece of a minimal surface. $\ddagger$ We shall prove that the isoperimetric inequality holds for every simply-connected rectifiable piece of every surface whose Gauss curvature $K$ is $\leqq 0$. This generalization is, in a way, final§; indeed, it is almost trivial (cf. \$2.7) that if a surface has the property that every simply-connected piece on it satisfies the isoperimetric inequality, then $K \leqq 0$ on the surface.

We shall make in our work the assumption, customary in differential

* See for instance A. R. Forsyth, Differential Geometry, Cambridge University Press, 1912, p. 84.

$\dagger$ See F. Riesz, Sur les fonctions subharmoniques et leur rapport à la théorie du potentiel (in two parts), Acta Mathematica, vol. 48 (1926), pp. 329-343, and vol. 54 (1930), pp. 321-360. We shall confine ourselves to the case of continuous subharmonic functions, though Riesz defines them more broadly. For a systematic treatment of the elementary properties of these functions, see P. Montel, Sur les fonctions convexes et les fonctions sousharmoniques, Journal de Mathématiques, (9), vol. 7 (1928), pp. 29-60.

$\ddagger$ T. Carleman, Zur Theorie der Minimalffüchen, Mathematische Zeitschrift, vol. 9 (1921), pp. $154-160$.

$\S$ That is, it is final in so far as the case of surfaces with $K \leqq 0$ is concerned. It is known, however, that for convex regions on a sphere with $K=K_{0}>0$ we have $a \leqq\left(l^{2}+K_{0} a^{2}\right) /(4 \pi)$. See F. Bernstein, $\ddot{U} b e r$ die isoperimetrische Eigenschaft des Kreises auf der Kugeloberfläche und in der Ebene, Mathematische Annalen, vol. 60 (1905), pp. 117-136. There are indications that perhaps the same inequality holds for simply-connected rectifiable regions on surfaces of constant negative curvature, $K=K_{0}<0$. The question arises then as to whether or not for all real $K_{0}$ the inequality $a \leqq\left(l^{2}+K_{0} a^{2}\right)$ $/(4 \pi)$ characterizes surfaces with variable curvature $K \leqq K_{0}$. 
geometry, that the surfaces and curves to be considered are analytic. This obviously unnecessary assumption serves the twofold purpose of avoiding certain unessential complications which would obscure the unity and simplicity of the method, and of dodging, certain essential difficulties which seem to require a thorough and presuriably interesting study.

Besides the isoperimetric inequality, we shall discuss briefly a few theorems which have been first proved for conformal maps of plane regions, have then been extended to conformal maps of minimal surfaces, and will be shown in this paper to hold for conformal maps of surfaces with $K \leqq 0$.

0.4. The following notation will simplify our next statement. If $g(u, v)$ is a continuous function in a domain $D$, we shall put

$$
\begin{aligned}
& A\left(g ; u_{0}, v_{0} ; \rho\right)=\frac{1}{\pi \rho^{2}} \iint_{\xi^{+}+\eta^{2}<\rho^{2}} g\left(u_{0}+\xi, v_{0}+\eta\right) d \xi d \eta, \\
& L\left(g ; u_{0}, v_{0} ; \rho\right)=\frac{1}{2 \pi} \int_{0}^{2 \pi} g\left(u_{0}+\rho \cos \phi, v_{0}+\rho \sin \phi\right) d \phi,
\end{aligned}
$$

where $\left(u_{0}, v_{0}\right)$ is the center and $\rho$ is the radius of a circular disc $\kappa:\left(u-u_{0}\right)^{2}$ $+\left(v-v_{0}\right)^{2} \leqq \rho^{2}$ which is comprised in $D$.

An important inequality, due to Carleman, ${ }^{*}$ can be stated then as follows: if $f(w)$ is an analytic function of $w=u+i v$ in $D$, then

$$
\left[A\left(|f|^{2} ; u_{0}, v_{0} ; \rho\right)\right]^{1 / 2} \leqq L\left(|f| ; u_{0}, v_{0} ; \rho\right)
$$

for every circular disc comprised in $D$. We shall show that a function $g(u, v)$, continuous and $\geqq 0$ in $D$, satisfies the inequality

$$
\left[A\left(g^{2} ; u_{0}, v_{0} ; \rho\right)\right]^{1 / 2} \leqq L\left(g ; u_{0}, v_{0} ; \rho\right)
$$

for every circular disc comprised in $D$, if and only if $\log g(u, v)$ is subharmonic in the part of $D$ where $g(u, v)>0$.

We shall use in this paper, as we did in a previous one, $\dagger$ the term function of class $P L$, meaning a function $g(u, v)$ continuous and $\geqq 0$, and such that $\log g(u, v)$ is subharmonic wherever $g(u, v)>0$. Then the above inequality (3) expresses a characteristic property of functions of class $P L$. On account of the formula (1) for $K$, this analytic fact is then readily seen to be equivalent to the geometric fact that the isoperimetric inequality is characteristic for surfaces with negative curvature (as explained in $\$ 0.3$ ).

0.5. It is natural to ask what happens if we replace in (3) the exponents 2 and $1 / 2$ by $\beta$ and $1 / \beta$ respectively, where $\beta$ is any real number. At the end of $\S 1$ we shall make a few very incomplete remarks concerning this question.

* Mathematische Zeitschrift, loc. cit.

† Subharmonic functions and minimal surfaces, these Transactions, vol. 35 (1933), pp. 648-661. 


\section{A Characterization of functions of Class $P L^{*}$}

1.1. The familiar example of a function of class $P L$ is the absolute value of an analytic function $f(w)$ of the complex variable $w=u+i v$. Indeed, as is well known, $\log |f(w)|$ is a harmonic function of $u$ and $v$, that is to say, $\Delta \log |f(w)|=0$.

We have the following theorem, due to Carleman. $\dagger$

If $f(w)$ is continuous in the unit circle $|w| \leqq 1$ and analytic in $|w|<1$, then

$$
\iint_{u^{2}+v<<1}|f(w)|^{2} d u d v \leqq \frac{1}{4 \pi}\left[\int_{0}^{2 \pi}\left|f\left(e^{i \phi}\right)\right| d \phi\right]^{2} .
$$

The sign of equality in (4) holds if and only if $f(w)=F^{\prime}(w)$, where $F(w)$ is a linear function

regular in $|w| \leqq 1$.

$$
\frac{a w+b}{c w+d}
$$

1.2. If we write the inequality (4) of Carleman as we did in 0.4 , then there arises the following question. Given a domain $D$ in the $u v$-plane, we ask for all functions $g(u, v)$, which are continuous and $\geqq 0$ in $D$, and satisfy the inequality (3) for every point $\left(u_{0}, v_{0}\right)$ in $D$ and for every $\rho$ such that the circular disc $\left(u-u_{0}\right)^{2}+\left(v-v_{0}\right)^{2} \leqq \rho^{2}$ is comprised in $D$. We shall prove the following

LEMMA. A function $g(u, v)$, continuous and $\geqq 0$ in a domain $D$, satisfies the inequality

$$
\left[A\left(g^{2} ; u_{0}, v_{0} ; \rho\right)\right]^{1 / 2} \leqq L\left(g ; u_{0}, v_{0} ; \rho\right)
$$

for every point $\left(u_{0}, v_{0}\right)$ in $D$ and for every $\rho$, such that the circular disc $\left(u-u_{0}\right)^{2}$ $+\left(v-v_{0}\right)^{2} \leqq \rho^{2}$ is comprised in $D$, if and only if $g(u, v)$ is of class $P L$ in $D$.

1.3. Let us first prove that if $g(u, v)$ is of class $P L$ in $D$, then the inequality (5) is satisfied. Suppose first that $g(u, v)>0$ in $D$. Consider any circular disc $\kappa$, comprised in $D$, with center $\left(u_{0}, v_{0}\right)$ and radius $\rho$. Denote by $C$ the perimeter of $\kappa$. Put

$$
\log g(u, v)=\phi(u, v)
$$

then by assumption $\phi(u, v)$ is subharmonic. Let $h(u, v)$ be the harmonic function in $\kappa$ coinciding with $\phi(u, v)$ on $C$. Then $\phi(u, v) \leqq h(u, v)$ in $\kappa \ddagger$, that is, $g(u, v) \leqq e^{h(u, v)}$ in $\kappa$. Consequently,

* For the precise definition and a discussion of elementary facts concerning these functions, see the authors' paper just cited.

$\dagger$ Mathematische Zeitschrift, loc. cit.

$\ddagger$ This is a general relation between a subharmonic function and a dominating harmonic function. See F. Riesz, Acta Mathematica, loc. cit., first part, p. 331. 


$$
\left[A\left(g^{2} ; u_{0}, v_{0} ; \rho\right)\right]^{1 / 2} \leqq\left[A\left(e^{2 h} ; u_{0}, v_{0} ; \rho\right)\right]^{1 / 2} .
$$

Also, $g(u, v)=e^{h(u, v)}$ on $C$, so that

$$
L\left(e^{h} ; u_{0}, v_{0} ; \rho\right)=L\left(g ; u_{0}, v_{0} ; \rho\right) .
$$

Let $h^{*}(u, v)$ be the conjugate harmonic function of $h(u, v)$. Then $f(w)$ $=e^{h+i h^{*}}$ is an analytic function of $w=u+i v$, and $|f(w)|=e^{h(u, v)}$. By Carleman's inequality (2) then

$$
\left[A\left(e^{2 h} ; u_{0}, v_{0} ; \rho\right)\right]^{1 / 2} \leqq L\left(e^{h} ; u_{0}, v_{0} ; \rho\right) .
$$

(5) follows from (6), (7), and (8).

Suppose now only that the function $g(u, v)$ of class $P L$ is $\geqq 0$ in $D$. Consider $g(u, v)+\epsilon$, where $\epsilon$ is a constant $>0$. Then $g(u, v)+\epsilon$ is $>0$ and of class $P L . \dagger$ Accordingly, the above discussion can be applied to $g(u, v)+\epsilon$, so that (5) holds for this function. As $g(u, v)$ is the uniform limit of $g(u, v)+\epsilon$ as $\epsilon \rightarrow 0$, we have (5) for a general $g(u, v)$ of class $P L$.

1.4. We shall show now that if $g(u, v)$ is a non-negative function defined and continuous in $D$, and if for every circular disc $\kappa$ comprised in $D$, the inequality (5) holds, then $g(u, v)$ is of class $P L$.

Suppose first that $g(u, v)$ has continuous derivatives of the first and second order, and let these derivatives be denoted by their standard symbols $p, q, r$, $s, t$. We assume for convenience that the point $\left(u_{0}, v_{0}\right)$ under discussion is $(0$, 0 ) and denote by $p_{0}$, etc., the value of $p$, etc., at $(0,0)$. Finally we shall denote by $\sigma_{j}$ certain quantities such that $\sigma_{j} / \rho^{2} \rightarrow 0$ as $\rho \rightarrow 0$, where $\rho^{2}=u^{2}+v^{2}$.

We have then, by the finite Taylor expansion,

so that

$$
\begin{aligned}
g(u, v)= & g_{0}+p_{0} u+q_{0} v+\frac{1}{2}\left(r_{0} u^{2}+2 s_{0} u v+t_{0} v^{2}\right)+\sigma_{1} \\
= & g_{0}+\left(p_{0} \cos \phi+q_{0} \sin \phi\right) \rho \\
& +\frac{1}{2}\left(r_{0} \cos ^{2} \phi+2 s_{0} \cos \phi \sin \phi+t_{0} \sin ^{2} \phi\right) \rho^{2}+\sigma_{1}, \\
g(u, v)^{2}= & g_{0}{ }^{2}+2 g_{0}\left(p_{0} \cos \phi+q_{0} \sin \phi\right) \rho \\
& +\left[\left(p_{0}{ }^{2}+g_{0} r_{0}\right) \cos ^{2} \phi+2\left(p_{0} q_{0}+g_{0} s_{0}\right) \cos \phi \sin \phi\right. \\
& \left.+\left(q_{0}{ }^{2}+g_{0} t_{0}\right) \sin ^{2} \phi\right] \rho^{2}+\sigma_{2},
\end{aligned}
$$

$$
\begin{aligned}
L(g ; 0,0 ; \rho) & =\frac{1}{2 \pi} \int_{0}^{2 \pi} g(\rho \cos \phi, \rho \sin \phi) d \phi=g_{0}+\frac{1}{4} \rho^{2}\left(r_{0}+t_{0}\right)+\sigma_{3}, \\
{[L(g ; 0,0 ; \rho)]^{2} } & =g_{0}^{2}+\frac{1}{2} \rho^{2} g_{0}\left(r_{0}+t_{0}\right)+\sigma_{4},
\end{aligned}
$$

$\dagger$ For the fact that the sum of two functions of class $P L$ is again a function of class $P L$, see the authors' paper in vol. 35 of these Transactions, pp. 648-661, $\$ 1.10$. 


$$
\begin{aligned}
A\left(g^{2} ; 0,0 ; \rho\right) & =\frac{1}{\pi \rho^{2}} \int_{0}^{\rho} \tau d \tau \int_{0}^{2 \pi} g(\tau \cos \phi, \tau \sin \phi)^{2} d \phi \\
& =g_{0}^{2}+\frac{1}{4} \rho^{2}\left[\left(p_{0}^{2}+q_{0}^{2}\right)+g_{0}\left(r_{0}+t_{0}\right)\right]+\sigma_{5} .
\end{aligned}
$$

By assumption, then,

$$
g_{0}^{2}+\frac{1}{4} \rho^{2}\left[\left(p_{0}^{2}+q_{0}^{2}\right)+g_{0}\left(r_{0}+t_{0}\right)\right]+\sigma_{5} \leqq g_{0}^{2}+\frac{1}{2} \rho^{2} g_{0}\left(r_{0}+t_{0}\right)+\sigma_{4},
$$

or

$$
\left(p_{0}^{2}+q_{0}^{2}\right)-g_{0}\left(r_{0}+t_{0}\right) \leqq 4\left(\sigma_{4}-\sigma_{5}\right) / \rho^{2} .
$$

The right-hand member of this last inequality $\rightarrow 0$ as $\rho \rightarrow 0$, so that the left-hand member is $\leqq 0$. Since any point of $D$ can be taken as $\left(u_{0}, v_{0}\right)$, we have then

$$
g(r+t)-\left(p^{2}+q^{2}\right) \geqq 0
$$

in $D$. Hence $g(u, v)$ is of class $P L$, since by computation (cf. $\S 0.2$ )

wherever $g>0$.

$$
\Delta \log g=\frac{g(r+t)-\left(p^{2}+q^{2}\right)}{g^{2}},
$$

1.5. Suppose now $\dagger$ that $g(u, v)$ has continuous derivatives of only the first order, but otherwise satisfies the conditions of $\$ 1.4$. For a small fixed $\tau>0$, put

$$
g(u, v ; \tau)=\frac{1}{\pi \tau^{2}} \iint_{\xi^{2}+\eta^{2}<\tau} g(u+\xi, v+\eta) d \xi d \eta .
$$

(Of course $g(u, v ; \tau)$ can be defined thus for only a subdomain $D^{\prime}$ of $D$, but this is of no consequence since $\tau$ is arbitrarily small.) That this function $g(u, v ; \tau)$ also satisfies (5) follows from Minkowski's inequality. $\ddagger$ Furthermore $g(u, v ; \tau)$ has continuous derivatives of the second order. $\S$ Hence $g(u, v ; \tau)$

$\dagger$ The assumptions of $\$ 1.4$ are sufficient for the applications to differential geometry which we shall make in $\S 2$, so that the reader interested primarily in those applications can omit $\$ 1.5$ and $\$ 1.6$ without loss of continuity in the discussion.

$\ddagger$ The necessary inequality follows, by a familiar passage to the limit, from the inequality

$$
\left(\sum_{k=1}^{n}\left(\sum_{j=1}^{m} a_{k j}\right)^{2}\right)^{1 / 2} \leqq \sum_{j=1}^{m}\left(\sum_{k=1}^{n} a_{k j}^{2}\right)^{1 / 2}
$$

which has the geometrical significance that the length of a polygonal line is at least as great as that of the line segment joining its end points.

$\S$ Concerning the properties and applications of this approximation by integral means, see E. Levi, Sopra una proprietd caratteristica delle funzione armoniche, Atti della Reale Accademia dei Lincei, vol. 18 (1909), pp. 10-15; H. E. Bray, Proof of a formula for an area, Bulletin of the American Mathematical Society, vol. 29 (1923), pp. 264-270; T. Rad6, Remarque sur les fonctions subharmoniques, Paris Comptes Rendus, vol. 186, pp. 346-348; T. Rad6, Sur le calcul de l'aire des surfaces courbes, Fundamenta Mathematicae, vol. 10 (1927), pp. 197-210; F. Riesz, loc. cit., second part, pp. 342-345. 
satisfies all the conditions of $\S 1.4$ and so is of class $P L$. Since $g(u, v ; \tau) \rightrightarrows g(u, v)$ as $\tau \rightarrow 0$ it follows that $g(u, v)$ is of class $P L$.

1.6. Suppose finally that $g(u, v)$ is only continuous, but otherwise satisfies the conditions of $\$ 1.4$. Then $g(u, v ; \tau)$, defined as above, has continuous first derivatives $\dagger$, and hence it satisfies the assumptions of $\$ 1.5$. According to $\S 1.5, g(u, v ; \tau)$ is of class $P L$ and consequently its uniform limit $g(u, v)$ is of class $P L$.

1.7. With regard to an application which we shall make in $\$ 2$, we need a slight (and incomplete) discussion of the sign of equality in (5). Suppose that $g(u, v)$ is continuous and positive in $\left(u-u_{0}\right)^{2}+\left(v-v_{0}\right)^{2} \leqq \rho^{2}$ and that $g(u, v)$ is of class $P L$ in $\left(u-u_{0}\right)^{2}+\left(v-v_{0}\right)^{2}<\rho^{2}$. Suppose that

$$
\left[A\left(g^{2} ; u_{0}, v_{0} ; \rho\right)\right]^{1 / 2}=L\left(g ; u_{0}, v_{0} ; \rho\right) .
$$

Then $g(u, v) \equiv\left|F^{\prime}(w)\right|$, where $F(w)$ is a linear function

$$
\frac{a w+b}{c w+d}
$$

which is regular in $\left|w-w_{0}\right| \leqq \rho$ and which does not reduce to a constant.

Indeed, if we go through the discussion in $\$ 1.3$, we find that in order to have (9), we must have (with the notations of $\$ 1.3$ )

$$
g(u, v) \equiv|f(w)|,
$$

where $f(w)$ satisfies the inequality (4) of Carleman with the sign of equality. On account of the theorem of Carleman, we have then $f(w)=F^{\prime}(w)$, where $F(w)$ has the desired form. This $F(w)$ cannot reduce to a constant at present, since then it would follow that $g(u, v) \equiv\left|F_{0}^{\prime}(w)\right|=0$, while we supposed that $g(u, v)>0$ throughout.

1.8. The question arises as to the significance of the inequality (5) if we replace the exponent 2 by a general (real) exponent $\beta$. The case $\beta=1$ can be settled easily; the reasoning used above in the case $\beta=2$ applies directly. $\neq$ There follows

A function $g(u, v)$, continuous in a domain $D$, satisfies there the inequality

$$
A\left(g ; u_{0}, v_{0} ; \rho\right) \leqq L\left(g ; u_{0}, v_{0} ; \rho\right)
$$

for every point $\left(u_{0}, v_{0}\right)$ in $D$ and for every $\rho$, such that the circular disc $\left(u-u_{0}\right)^{2}$ $+\left(v-v_{0}\right)^{2} \leqq \rho^{2}$ is comprised in $D$, if and only if $g(u, v)$ is subharmonic in $D$.

1.9. For values of $\beta$ other than 1 and 2 , the method of $\$ 1.4, \S 1.5, \S 1.6$ yields theorems whose statements vary according to the location of $\beta$ with

† See third footnote on p. 667.

$\ddagger$ Actually, though, there is a much simpler way of handling the case $\beta=1$. 
respect to the special values $0,1,2$. By way of illustration, we mention the following statements.

Suppose $g(u, v)$ is continuous and $\geqq 0$ in a domain $D$. Suppose that for a certain exponent $\beta$ the inequality

$$
\left[A\left(g^{\beta} ; u_{0}, v_{0} ; \rho\right)\right]^{1 / \beta} \leqq L\left(g ; u_{0}, v_{0} ; \rho\right)
$$

holds for every point $\left(u_{0}, v_{0}\right)$ in $D$ and for every $\rho$ such that the circular disc $\left(u-u_{0}\right)^{2}+\left(v-v_{0}\right)^{2} \leqq \rho^{2}$ is comprised in $D$.

If $1<\beta<2$, then it follows that $g^{2-\beta}$ is subharmonic in $D$.

If $\beta>2$, it follows that $1 / g^{\beta-2}$ is superharmonic.

In a general way, the greater $\beta$, the stronger the inference will be as to the subharmonic character of $g(u, v)$. For $\beta<1, g(u, v)$ need not be subharmonic.

For $\beta=1$ and $\beta=2$ the inequality (10) has been shown, in what precedes, to be a necessary and sufficient criterion for a certain subharmonic property. An equally complete discussion for a general exponent might lead to interesting questions.

\section{Applications to SURFACES OF NEgATIVE CURVATURE}

2.1. Let there be given a piece of surface $S$ in a representation

$$
S: x=x(u, v), y=y(u, v), z=z(u, v), u^{2}+v^{2} \leqq \rho^{2},
$$

with the following properties.

(a) $x(u, v), y(u, v), z(u, v)$ and their first partial derivatives are continuous in $u^{2}+v^{2} \leqq \rho^{2}$.

(b) In $u^{2}+v^{2}<\rho^{2}, x(u, v), y(u, v), z(u, v)$ have continuous partial derivatives of the third order.

(c) The representation (11) is isothermic, that is to say, $E=G, F=0$, in $u^{2}+v^{2} \leqq \rho^{2}$. We put $E=G=\lambda(u, v)$. Then $\lambda \geqq 0$; but we suppose that $\lambda>0$ in $u^{2}+v^{2} \leqq \rho^{2}$.

2.2. Lemma. If the Gauss curvature $K$ of the surface $S$, given in a representation as described in $\$ 2.1$, is $\leqq 0$, then the area and the perimeter $l$ of $S$ satisfy the inequality $a \leqq l^{2} /(4 \pi)$. The sign of equality holds if and only if $K \equiv 0$, and $S$ is a geodesic circle (that is to say, $S$ is a developable and there exists a point $O$ on $S$ such that the geodesic distance of $O$ from every point of the perimeter of $S$ is. the same).

The proof is as follows. With the notation of $\$ 0.4$ we have

$$
a=\pi \rho^{2} A(\lambda ; 0,0 ; \rho), l=2 \pi \rho L\left(\lambda^{1 / 2} ; 0,0 ; \rho\right) .
$$

On account of the assumption $K \leqq 0$, the function $\lambda(u, v)$ is of class $P L$ (see 
$\S 0.2$ and the definition of functions of class $P L)$. Hence $\dagger$ the function $\lambda(u, v)^{1 / 2}$ is also of class $P L$. From $\S 1.2$ it follows, therefore, for $g=\lambda^{1 / 2}$, that

$$
[A(\lambda ; 0,0 ; \rho)]^{1 / 2} \leqq L\left(\lambda^{1 / 2} ; 0,0 ; \rho\right) .
$$

The inequality $a \leqq l^{2} /(4 \pi)$ follows now immediately from (12) and (13).

Suppose now that we have $a=l^{2} /(4 \pi)$. Then we must have the sign of equality in (13). Consequently (see $\$ 1.7$ ) we have

$$
\lambda(u, v)^{1 / 2}=\left|F^{\prime}(w)\right|,
$$

where $F(w)$ has the form $(a w+b) /(c w+d)$, and $F(w)$ is regular and not constant in $|w| \leqq \rho$. Hence the equation $w^{*}=F(w)$ carries the circle $|w| \leqq \rho$ in a one-to-one and conformal way into a certain circular disc $\kappa^{*}$ in the $w^{*}=u^{*}+i v^{*}$ plane. Introducing $u^{*}, v^{*}$ as new parameters, we obtain the equations of $S$ in the form

$$
S: x=\xi\left(u^{*}, v^{*}\right), y=\eta\left(u^{*}, v^{*}\right), z=\zeta\left(u^{*}, v^{*}\right),\left(u^{*}, v^{*}\right) \text { in } \kappa^{*} .
$$

Since we passed from the isothermic parameters $u, v$ to the new parameters $u^{*}, v^{*}$ by a conformal map, it follows that $u^{*}, v^{*}$ are also isothermic parameters. Hence if we denote by $E^{*}, F^{*}, G^{*}$ the first fundamental quantities relative to the representation (15), we have $E^{*}=G^{*}, F^{*}=0$. If we put $E^{*}=G^{*}=\lambda^{*}\left(u^{*}, v^{*}\right)$, then we have, by simple computation,

$$
\lambda^{* 1 / 2}=\lambda^{1 / 2}\left|\frac{d w}{d w^{*}}\right|=\lambda^{1 / 2} /\left|F^{\prime}(w)\right|=1,
$$

on account of (14). Hence $E^{*}=G^{*}=1, F^{*}=0$. That is to say, the representation (15) is an isometric map of $S$ (every arc on $S$ has the same length as its image).

2.3. In order to apply the lemma of $\S 2.2$ to a given piece of surface, we have to represent the surface as required in $\$ 2.1$. Thus it is necessary to refer to existence theorems on conformal mapping, and the validity of the isoperimetric inequality $a \leqq l^{2} /(4 \pi)$ is made to depend upon the available results concerning the theory of conformal mapping. Since we are unable at this time to prove the most general statement which is likely to be true, we restrict ourselves to the following theorem which might be considered as perfectly general according to the usual standards in differential geometry.

2.4. THEOREM. Let there be given an analytic surface in the xyz-space, that is to say, a surface which admits, in the vicinity of every one of its points, a representation $x=x(u, v), y=y(u, v), z=z(u, v)$, where $x(u, v), y(u, v), z(u, v)$

$\dagger$ Every positive power of a function of class $P L$ is again a function of class $P L$; see the authors' paper in these Transactions, vol. 35, pp. 648-661, $\$ 1.8$. 
are analytic functions of $u$, v, and where $E G-F^{2}>0$. Denote by $K$ the Gauss curvature of the surface. Then $K \leqq 0$ is a necessary and sufficient condition that the area $a$ and the perimeter $l$ of every simply-connected portion, bounded by an analytic curve, of the surface satisfy the isoperimetric inequality $a \leqq l^{2} /(4 \pi)$.

2.5. To prove this theorem, suppose first that $K \leqq 0$. Let $S$ be any simplyconnected portion of the surface, bounded by an analytic curve. Take a simply-connected open portion $S^{*}$ of the surface, such that $S$ is interior to $S^{*}$. On account of general theorems, $S^{*}$ admits of an isothermic representation

$$
S^{*}: x=\xi\left(u^{*}, v^{*}\right), y=\eta\left(u^{*}, v^{*}\right), z=\zeta\left(u^{*}, v^{*}\right), u^{* 2}+v^{* 2}<1,
$$

where $E^{*}=G^{*}>0, F^{*}=0$, and $\xi, \eta, \zeta$ are analytic functions of $u^{*}, v^{*}$. The portion $S$ appears in this map as a Jordan region $R^{*}$ in $u^{* 2}+v^{* 2}<1$, bounded by an analytic Jordan curve $C^{*}$. We map then $R^{*}$ in a one-to-one and conformal way upon $u^{2}+v^{2} \leqq 1$; on account of the analyticity of $C^{*}$, this map remains analytic on $u^{2}+v^{2}=1$. Thus we obtain a map of $S$ as required in $\$ 2.1$, and then the lemma of $\$ 2.2$ gives the desired inequality $a \leqq l^{2} /(4 \pi)$.

2.6. $\dagger$ Suppose, conversely, that we have $a \leqq l^{2} /(4 \pi)$ for every portion $S$ of a surface as described in $\$ 2.4$. Take such a portion $S$. Applying the construction of $\$ 2.5$, we obtain for $S$ a representation

$$
S: x=x(u, v), y=y(u, v), z=z(u, v), u^{2}+v^{2} \leqq 1,
$$

with the properties required in $\$ 2.1$. If $\kappa:\left(u-u_{0}\right)^{2}+\left(v-v_{0}\right)^{2} \leqq \rho^{2}$ is any circular disc comprised in $u^{2}+v^{2}<1$, then there corresponds to $\kappa$, by means of (16), a portion $S_{0}$ whose area $a_{0}$ and perimeter $l_{0}$ satisfy by assumption the inequality $a_{0} \leqq l_{0}{ }^{2} /(4 \pi)$. If we use again the notation $E=G=\lambda(u, v)$, then

$$
a_{0}=\pi \rho^{2} A\left(\lambda ; u_{0}, v_{0} ; \rho\right), l_{0}=2 \pi \rho L\left(\lambda^{1 / 2} ; u_{0}, v_{0} ; \rho\right),
$$

and hence $a_{0} \leqq l_{0}{ }^{2} /(4 \pi)$ implies that

$$
\left[A\left(\lambda ; u_{0}, v_{0} ; \rho\right)\right]^{1 / 2} \leqq L\left(\lambda^{1 / 2} ; u_{0}, v_{0} ; \rho\right) .
$$

Since this holds for every circular disc $\left(u-u_{0}\right)^{2}+\left(v-v_{0}\right)^{2} \leqq \rho^{2}$ comprised in $u^{2}+v^{2}<1$, it follows (see $\left.\S 1.2\right)$ that $\lambda^{1 / 2}$ and consequently $\ddagger \lambda$ is of class $P L$ in $u^{2}+v^{2}<1$. Hence (see $\left.\S 0.2\right) K \leqq 0$ on $S$. Since $S$ was any portion of the given surface, this proves that $K \leqq 0$ on the whole surface.

2.7. The reasoning of $\$ 2.6$ can be replaced by the following argument. Take any point $O$ on the surface and denote by $S_{\rho}$ the portion of the surface which consists of the points of the surface within and on the geodesic circle

$\dagger$ A differential geometer will probably find the proof of $\$ 2.7$ preferable.

$\ddagger$ See footnote on p. 670 . 
with center $O$ and radius $\rho$. Then the area and perimeter of $S_{\rho}$ are functions of $\rho$ which admit of developments beginning as followst:

$$
\begin{aligned}
& a(\rho)=\pi \rho^{2}-\frac{1}{12} \pi K_{0 \rho^{4}}+\cdots, \\
& l(\rho)=2 \pi \rho-\frac{1}{3} \pi K_{0} \rho^{3}+\cdots,
\end{aligned}
$$

where $K_{0}$ is the Gauss curvature at the point $O$. We have then

or

$$
a(\rho)-\frac{1}{4 \pi} l(\rho)^{2}=\frac{1}{4} \pi K_{0} \rho^{4}+\cdots
$$

$$
K_{0}=\frac{4}{\pi} \lim _{\rho \rightarrow 0} \frac{a(\rho)-\frac{1}{4 \pi} l(\rho)^{2}}{\rho^{4}} .
$$

Since, by assumption, the numerator is $\leqq 0$, this proves that $K_{0} \leqq 0$. Since $O$ is any point on the surface, we have then $K \leqq 0$ on the whole surface.

2.8. Let us now consider the sign of equality in the isoperimetric inequality. In order to illustrate a very trivial point, let us consider a Jordan region in the plane, bounded by a rectifiable curve which is not a circle. Then we have $a<l^{2} /(4 \pi)$. Putting some hills on this plane region, we can keep the perimeter $l$ fixed and increase the area until we have $a=l^{2} /(4 \pi)$. Since our hills were otherwise quite arbitrary, it is clear that from $a=l^{2} /(4 \pi)$ alone we cannot conclude anything concerning the surface. On the other hand, if we restrict ourselves to analytic surfaces with $K \leqq 0$, and if we use the fact that $K \equiv 0$ on such a surface as soon as $K \equiv 0$ on any subregion, then the lemma of $\$ 2.2$ yields immediately the following result.

If an analytic surface with $K \leqq 0$ contains some portion for which the sign of equality holds in the isoperimetric inequality $a \leqq l^{2} /(4 \pi)$, then $K \equiv 0$ on the surface, and $a=l^{8} /(4 \pi)$ holds only for the geodesic circles.

2.9. In what precedes, we extended a theorem, previously proved only for minimal surfaces, to surfaces with $K \leqq 0$. A systematic study of similar generalizations might lead to interesting results. We mention here a few immediate facts.

Let $S$ be a piece of surface with $K \leqq 0$, which admits an isothermic representation

$$
S: x=x(u, v), y=y(u, v), z=z(u, v), u^{2}+v^{2} \leqq \rho^{2},
$$

with the properties described in $\$ 2.1$. Put again $E=G=\lambda(u, v)$, and suppose

† See for instance L. P. Eisenhart, Differential Geometry, Ginn and Company, 1909, p. 209. 
that $\lambda(0,0)=1$ (that is to say, that the linear magnification is unity at the origin). Denote by $l(r)$ the length of the image of $u^{2}+v^{2}=r^{2}$, and by $a(r)$ the area of the image of $u^{2}+v^{2}<r^{2}$. Then

(a) $l(r)$ is an increasing function of $r+$;

(b) $l(r) \geqq 2 \pi r \ddagger$;

(c) $a(r) \geqq \pi r^{2} . \ddagger$

We have

$$
l(r)=\int_{0}^{2 \pi} \lambda(r \cos \phi, r \sin \phi)^{1 / 2} r d \phi
$$

Since $K \leqq 0$, it follows $\S$ that $\lambda(u, v)^{1 / 2}$ is of class $P L$. Also (see $\$ 1.1$ ), $r=$ $|u+i v|$ is of class $P L$. Therefore $\| r \lambda^{1 / 2}$ is of class $P L$, and consequently $\pi r \lambda^{1 / 2}$ is subharmonic. (a) follows then from the above expression for $l(r)$ and from the fact that the integral mean of a subharmonic function is an increasing function of $r . \dagger \dagger$

To prove (b) and (c), observe that

$$
\begin{aligned}
& a(r)=\pi r^{2} A(\lambda ; 0,0 ; r), \\
& l(r)=2 \pi r L\left(\lambda^{1 / 2} ; 0,0 ; r\right) .
\end{aligned}
$$

On account of $K \leqq 0, \lambda$ and consequently $\lambda^{1 / 2}$ are subharmonic. Hence $\ddagger$

$$
\begin{aligned}
& 1=\lambda(0,0) \leqq A(\lambda ; 0,0 ; r) \\
& 1=\lambda(0,0)^{1 / 2} \leqq L\left(\lambda^{1 / 2} ; 0,0 ; r\right)
\end{aligned}
$$

Thus (b) and (c) follow from (18) and (19).

† For the plane case, see L. Bieberbach, Über die konforme Kreisabbildung nahezu kreisförmiger Bereiche, Berlin Sitzungsberichte, 1924, pp. 181-188; for minimal surfaces, see T. Rad6, Some remarks on the problem of Plateau, Proceedings of the National Academy of Sciences, vol. 16 (1930), pp. 242-248, and On Plateau's problem, Annals of Mathematics, vol. 31 (1930), pp. 457-469.

$\ddagger$ For the plane case, see L. Bieberbach, Palermo Rendiconti, vol. 38 (1914), pp. 98-112; for minimal surfaces, see E. F. Beckenbach, The area and boundary of minimal surfaces, Annals of Mathematics, vol. 33 (1932), pp. 658-664.

$\S$ See the authors' paper in these Transactions, vol. 35 , pp. $648-661, \S 1.8$.

\| The product of two functions of class $P L$ is again a function of class $P L$; see the authors' paper in these Transactions, vol. 35 , pp. 648-661, $\$ 1.8$.

T Every positive power of a function of class $P L$ is a subharmonic function; see the authors' paper in these Transactions, vol. 35 , pp. $648-661, \$ \$ 1.7$ and 1.8 .

†† See F. Riesz, Acta Mathematica, loc. cit., first part, p. 338.

$\ddagger$ These inequalities express two of several clearly equivalent definitions of subharmonic functions. See J. E. Littlewood, On the definition of a subharmonic function, London Mathematical Society Journal, vol. 2 (1927), pp. 189-192. 
COROLLARY. If the sign of equality holds in (b) or (c) for any value of $r$, $0<r \leqq \rho$, then $\lambda(u, v) \equiv 1$, so that (see \$2.2) the map (17) is isometric. In other words, $S$ is a developable piece of surface and is a geodesic circle given in isometric representation.

To see this, consider for instance the sign of equality in (c); then

$$
\lambda(0,0)=\frac{1}{\pi r^{2}} \iint_{u \text { \& }+v<<r 2} \lambda(u, v) d u d v .
$$

Consequently $\lambda(0,0)=h(0,0)$, where $h(u, v)$ is the harmonic function in $u^{2}+v^{2}<r^{2}$ coinciding with $\lambda(u, v)$ on $u^{2}+v^{2}=r^{2}$, and therefore $\dagger \lambda(u, v)$ $\equiv h(u, v)$, so that

$$
\Delta \lambda(u, v)=0 .
$$

But $\lambda(u, v)$ is also of class $P L$, so that (see $\$ 0.2$ )

$$
\lambda \Delta \lambda-\left(\lambda_{u}{ }^{2}+\lambda_{v}{ }^{2}\right) \geqq 0 .
$$

Consequently $\lambda_{u}{ }^{2}+\lambda_{v}{ }^{2} \leqq 0$ and therefore $\lambda(u, v)$ is constant. But $\lambda(0,0)=1$, so that $\lambda(u, v) \equiv 1$. The same argument holds for the sign of equality in (b), with $\lambda(u, v)^{1 / 2}$ in place of $\lambda(u, v)$.

$\dagger \lambda(u, v)$ is subharmonic, and therefore, by the definition of subharmonic functions (see F. Riesz, Acta Mathematica, loc. cit., first part, p. 331) $\psi(u, v)=\lambda(u, v)-h(u, v)$ is subharmonic. We have $\psi(r \cos \phi, r \sin \phi) \equiv 0, \psi(0,0)=0$. But a subharmonic function cannot attain an interior maximum unless it is identically constant (see above reference to F. Riesz, p. 331). Therefore $\psi(u, v) \equiv 0$, $\lambda(u, v) \equiv h(u, v)$.

Ohio State University, Columbus, OHio 